\title{
Les organismes communautaires au Québec : De la coexistence à la supplémentarité
}

\author{
Sébastien Savard \\ Université d'Ottawa
}

\author{
Jean Proulx \\ Université du Québec à Montréal
}

\section{RÉSUMÉ}

Cet article présente les résultats d'une recherche effectuée auprès de 52 organismes du tiers secteur québécois sur la question des relations qu'ils entretiennent avec un partenaire du secteur public. Les objectifs de la recherche étaient doubles : 1) élaborer un outil d'autoévaluation validé des relations entre organismes du tiers secteur et l'État et 2) procéder à une analyse transversale des informations produites par les 52 organismes du tiers secteur ayant participé à la validation de l'outil d'autoévaluation. Les résultats démontrent que les relations entre les deux groupes d'acteurs se sont stabilisées et même améliorées depuis vingt ans. Cependant, la contribution des organismes du tiers secteur est encore largement orientée vers la coproduction de services publics davantage que sur la coconstruction de politiques sociales destinées à une population commune.

\section{ABSTRACT}

This article presents the results of a study involving 52 nonprofit organizations in Quebec focused on their relationships with public-sector partners. The objectives of the study were twofold: 1) to develop and validate a self-evaluation tool to assess relationships between third-sector organizations and the State and 2) to conduct a transversal analysis of information obtained from the 52 nonprofit organizations that took part in the validation of the self-evaluation tool. The results show that relationships between the two categories of stakeholders stabilized and even improved over the previous twenty years. However, the contribution of nonprofit organizations is still largely oriented toward the coproduction of public services rather than the shared construction of social policies for a common population.

\section{Mots clés / Keywords :}

Relations; Organismes communautaires; OSBL; Secteur public; État; Partenariat; Services sociaux; Outil d'autoévaluation Québec / Relationships; Community organizations; NPOs; Public sector; State; Partnership; Social services; Self-evaluation tool Quebec 


\section{INTRODUCTION}

Depuis le début des années 1980, au Québec comme ailleurs, nous avons assisté à un nouveau partage des responsabilités entre l'État et les acteurs de la société civile dans la réponse aux besoins sociaux et économiques de la population. C'est ainsi que le tiers secteur a été appelé, au cours des trente dernières années, à s'inscrire dans une nouvelle relation dite « partenariale » avec l'État.

Au Québec, le tiers secteur est aujourd'hui présent dans une multitude de secteurs d'activité allant des services à la petite enfance à la formation de la main d'œuvre et à l'employabilité. C'est cependant dans le secteur de la santé et des services sociaux que les organismes communautaires sont de loin les plus nombreux. En 1990, la réforme Côté venait en effet les reconnaître officiellement dans la Loi 120, ce qui a fortement contribué à leur développement. Par ailleurs, la réforme Couillard de 2003 invitait les centres de santé et de services sociaux (CSSS) nouvellement créés à conclure, dans le cadre de leur « responsabilité populationnelle ", des ententes de services avec leurs partenaires pour la livraison de services, y compris les organismes communautaires. C'est donc vers une certaine forme de contractualisation que cette réforme tend à vouloir faire évoluer les relations entre ces deux acteurs.

Cette nouvelle relation entre les acteurs publics et ceux du tiers secteur peut prendre, plusieurs configurations selon le pays, et aussi à l'intérieur d'un même État. Dans certains cas, elle peut être empreinte d'une forte dépendance des organismes du tiers secteur vis-à-vis de l'État, tandis que dans d'autres on peut assister à un rapport qui soit plus égalitaire et dans lequel l'État voit dans les organismes du tiers secteur de véritables partenaires. Un certain nombre d'études ont cherché à caractériser les relations entre l'État et le tiers secteur au Québec (Panet-Raymond et Bourque, 1991; White et al. 1992; Vaillancourt, 1997), mais peu ont procédé à l'analyse des relations dans plusieurs secteurs d'intervention à partir d'une méthodologie permettant aux représentants d'organismes de se prononcer sur le modèle de relation qu'ils établissent avec l'État. C'est ce que nous avons voulu réaliser dans le cadre de cette recherche.

Dans le présent article, nous ferons part des résultats d'une recherche que nous avons menée auprès de 52 organismes du tiers secteur qui sont en relation avec des établissements ou organismes publics. Ces 52 organismes viennent de partout au Québec et interviennent dans des secteurs d'activité variés. Cette recherche s'est inscrite dans la poursuite d'une recherche antérieure au cours de laquelle nous avions identifié six types de rapport possibles entre un organisme du tiers secteur et un établissement ou un organisme public (Proulx, Bourque et Savard, 2007). Elle été réalisée à partir d'un outil que nous avons construit, lequel visait justement à permettre aux organismes d'identifier la nature de leur relation avec un établissement ou un organisme public donné.

\section{PROBLÉMATIQUE DE RECHERCHE}

Un peu partout dans le monde, l'actualisation des politiques publiques se fait avec un concours grandissant des organismes du tiers secteur'1. Désormais, l'État n'a plus le monopole de l'offre de services d'intérêt public, mais la partage à des degrés divers avec d'autres acteurs, qu'il s'agisse du secteur privé à but lucratif, des familles et des proches de personnes nécessitant différentes formes de soins, de soutien ou d'aide, ou encore d'organismes du tiers secteur.

Le " partenariat» est donc devenu un thème récurrent dans les politiques publiques. En fait, depuis la fin des années 1970, il est au cœur des stratégies de la plupart des pays occidentaux qui, confrontés à la fois à 
une crise financière et à une crise "des résultats » (Groulx, 1993), ont commencé à revoir leur mode de gestion du social fondé sur un nouveau partage des responsabilités avec les acteurs de la société civile. Selon Proulx, ce nouveau «mode de gestion du social» repose essentiellement sur quatre axes: la personne au centre des préoccupations; une tendance à la régionalisation et au local; l'appel aux ressources de la communauté (les familles, les proches, les organismes communautaires); et le partenariat comme stratégie privilégiée d'intervention (Proulx, 1997, p. 27).

Ce changement de cap dans la gouvernance de l'État est attribuable autant aux critiques provenant de la gauche que de la droite. En effet, d'un côté, les tenants de l'idéologie néolibérale revendiquent un État moindre sous prétexte que celui-ci coûte cher et que le secteur privé s'avère un agent économique plus efficace. La gauche de son côté, sans remettre en cause le bien-fondé de l'État-Providence, dénonce ses effets pervers, notamment la lourde bureaucratie qu'il a produite ainsi qu'une perte de « lien social » dans une sorte de "solidarité mécanique " (Rosanvallon, 1981), revendiquant ainsi des services plus souples et plus légers et un rôle accru pour les réseaux de soutien naturels (Groulx, 1993).

Mais il n'y a pas unanimité sur le sens à donner à ce changement. Si certains y voient une occasion pour l'État québécois de se départir de certaines responsabilités pour les confier aux organismes du tiers secteur dans une relation de sous-traitance, d'autres y voient plutôt une « nouvelle forme de pratique sociale qui s'appuie sur une redéfinition des rapports entre l'État et la société civile dans le but d'introduire des réformes et de démocratiser certains secteurs d'activités » (Bilette et al. 1995, p. 4). Caillouette et coll. parlent quant à eux d'un nouveau " paradigme », d'une nouvelle forme de " gouvernance », c'est-à-dire de "l'instauration de nouveaux modes d'élaboration des politiques publiques » qui incluraient les acteurs de la société civile (Caillouette et al, 2007, p. 452), ce que Vaillancourt appelle la «coconstruction» des politiques publiques (Vaillancourt, 2008).

\section{LA SITUATION AU QUÉBEC}

Le Québec ne fait pas exception à la règle. Ainsi, aujourd'hui, le tiers secteur est présent dans une multitude de champs d'activité : services de garde à l'enfance; logement social; santé et services sociaux; services d'aide à l'emploi; développement local, etc. En fait, comme le soulignent René et Gervais, « Ce qui étonne devant l'ampleur du phénomène, ce n'est pas tant le nombre de secteurs investis que la pénétration $\mathrm{du}$ paradigme à tous les niveaux de la vie en société » (René et Gervais, 2001, p. 23). Par ailleurs, le partenariat est une pratique « polymorphe qui peut s'exercer à plusieurs échelles : nationale, régionale et locale » (Bourque, 2007a, p. 298).

En somme, dans ce nouveau mode de gestion du social, non seulement le Québec ne fait-il pas exception, mais plusieurs croient même que le Québec se démarquerait par un modèle de développement qui lui serait propre (Bourque, 2000; Vaillancourt, 2012; Caillouette et coll., 2007; Favreau et Larose, 2007; Jetté, 2008), un modèle caractérisé notamment par un État "plus ouvert à la participation de la société civile ", des formes de gouvernance qui font de la place aux acteurs locaux et la participation des organismes du tiers secteur «non seulement à la mise en application mais aussi à la Coconstruction démocratique des politiques » (Vaillancourt, 2012, p. 127). Toutefois, depuis l'élection d'un gouvernement libéral en 2003, Caillouette et coll. (2007) soutiennent qu'il y a eu « rupture » dans ce modèle de développement.

Ainsi, avec la réingénierie de l'État, et notamment avec la réforme Couillard dans le domaine de la santé et des services sociaux, nous serions passés d'un modèle de régulation "partenariale " à un modèle de régulation «marchande », dans lequel l'État « n'est plus tenu à un dialogue avec les acteurs de la société 
civile ». Selon ces mêmes auteurs, "Dans cette perspective marchande, le seul interlocuteur de l'État est le secteur privé, qui comprend dans ce cas les organisations de la société civile, mais alors réduites à une identité de fournisseurs de biens et de services. » Bourque a aussi noté comment les Lois 25 et 83 viennent modifier le rapport des CSSS avec les organismes communautaires, ceux-ci s'inscrivant dorénavant dans un rapport plus hiérarchique dans lequel les CSSS pourront de surcroît sous-traiter avec les organismes communautaires pour la livraison de services (Bourque, 2004). Toutefois, dans le processus de production des projets cliniques auquel les CSSS sont tenus, autant Bourque (2007b) que Caillouette et coll. soutiennent que la réforme Couillard peut céder la place à des marges de manœuvre possibles, voire même à de la «coconstruction » dans certains cas (Bourque, 2007b), puisque «les cultures construites dans les décennies antérieures demeurent en partie effectives » (Caillouette et coll., 2007, p. 462).

\section{LES RAPPORTS PUBLIC / COMMUNAUTAIRES AU QUÉBEC}

C'est donc dans ce contexte qu'il faut appréhender la question des rapports entre les organismes du tiers secteur et les établissements et organismes publics. Au Québec, ceux-ci ont été largement étudiés dans les années 1980 et 1990, au plus fort de l'émergence de ce nouveau phénomène ${ }^{2}$. De la littérature, il ressort essentiellement que les relations entre les organismes communautaires sont pour le moins difficiles et souvent empreintes de tensions, et se caractérisent souvent par des rapports asymétriques et hiérarchiques (Proulx, 1997; Savard, 2002; Bourque, 2007a).

Les rapports public/communautaires ont été beaucoup moins étudiés depuis le début des années 2000. Les quelques recherches qui ont été faites l'ont été après 2003, dans la foulée de la réforme Couillard et des craintes parfois exprimées d'un assujettissement des organismes communautaires aux ententes de services avec les CSSS et à la sous-traitance. II est par ailleurs intéressant de constater que, par rapport aux recherches antérieures, les recherches qui ont été réalisées depuis 2003 apportent un éclairage différent. En effet, la plupart des recherches plus récentes montrent que les ententes de services ne sont pas une pratique courante et que, globalement, là où elles existent, elles n'ont pas modifié de façon déterminante l'interface entre les deux acteurs, et ce, que ce soit dans le secteur de l'aide à la famille et à la jeunesse (Bourque, 2007b; Savard et coll., 2008), dans le secteur de la perte d'autonomie liée au vieillissement (Bourque et coll., 2011; Savard et coll., 2010), dans le domaine du handicap (Proulx, 2011) ou encore dans le secteur de la santé et des services sociaux en général (Proulx et Boudreault, 2009). Ces mêmes recherches ont également démontré que les relations empruntent des modèles très variés (Bourque, 2007b) et que, en général, on ne peut pas parler d'un assujettissement des organismes communautaires aux règles et aux demandes du réseau public. Les recherches de Savard et coll. (2008 et 2010) montrent par ailleurs que l'on ne peut pas non plus parler d'une reconnaissance totale et complète des organismes communautaires, puisque la relation qu'ils ont établie avec les établissements publics serait plutôt de l'ordre de la coexistence, c'est-à-dire une relation dans laquelle les acteurs communautaires et publics collaborent sans pour autant que la relation soit contraignante et sans que les établissements publics cherchent à soutenir ou à encourager la contribution des organismes communautaires. 


\section{OBJECTIFS DE LA RECHERCHE ET CADRE D'ANALYSE}

C'est dans le contexte de la réingénierie de l'État et de la réforme Couillard que, en 2003, nous avons entrepris une recherche visant à approfondir la question de ce que nous avons appelé les interfaces entre l'État et les organismes du tiers secteur. Par cette recherche, nous souhaitions alimenter la réflexion chez les acteurs du terrain et les aider à se situer dans leurs rapports à l'État en fonction d'une diversité de modèles possibles.

Pour ce faire, nous avons procédé à une revue de littérature internationale portant sur différentes typologies existantes. Notre choix s'est arrêté sur la typologie développée par Jennifer M. Coston (1998), typologie qui, au terme de la recherche, a été adaptée au contexte québécois (Proulx, Bourque et Savard, 2007). II a été possible d'identifier la présence éventuelle de six types de rapport entre les établissements publics et les organismes du tiers secteur : le rapport de compétition; le rapport de sous-traitance; le rapport de tiers-parti; le rapport de coexistence; le rapport de supplémentarité; et le rapport de coconstruction (Proulx, Bourque et Savard, 2007). Ces types de rapport sont définis de la façon suivante :

- Le rapport de "compétition ». Dans le rapport de compétition, l'établissement ou l'organisme public n'est pas favorable à la présence d'organismes du tiers secteur dans son champ d'activité. Les deux types d'organisation sont donc en compétition sur le terrain pour l'exercice de certaines activités, mais aussi pour l'obtention du financement lié à ces activités.

- Le rapport de "sous-traitance ». Dans le rapport de sous-traitance, l'établissement ou l'organisme public est l'acteur central responsable de la planification et de l'organisation des services, mais délègue la livraison de certains services à des organismes du tiers secteur pour une clientèle dont il conserve toutefois la responsabilité (l'imputabilité). Ce type de rapport fait habituellement l'objet d'une entente écrite liant les deux organisations qui traduit avec précision les engagements des deux partis.

- Le rapport de « tiers parti ». Le rapport de tiers parti se rapproche du rapport de soustraitance. II s'en distingue par le fait que l'établissement ou l'organisme public se limite ici à établir les orientations et les priorités, et délègue aux organismes du tiers secteur la responsabilité d'organiser la production des services, limitant ainsi son influence sur les activités de l'organisme et sur l'utilisation des fonds. Dans ce type de rapport, l'établissement ou l'organisme public délègue aussi sa responsabilité à l'égard de la clientèle (son imputabilité).

- Le rapport de «coexistence ». Dans le rapport de coexistence, l'établissement ou l'organisme public est « sympathique » à l'égard des activités des organismes du tiers secteur dans un champ d'activité donné, mais n'est pas nécessairement proactif pour les soutenir. Le rapport se limite souvent à des échanges d'information dans une "coexistence non contraignante ».

- Le rapport de "supplémentarité ». Dans le rapport de supplémentarité, l'établissement ou l'organisme public met en place des services publics en fonction des "préférences moyennes" de la population, mais accepte de soutenir en partie les "préférences minoritaires » prises en charge par les organismes du tiers secteur. Ceux-ci répondent donc à une demande laissée vacante par les services publics, et il y a reconnaissance mutuelle du rôle joué par les deux types d'organisation. Les organismes du tiers secteur peuvent également être mis à contribution pour l'élaboration des politiques et des programmes publics.

- Le rapport de «Coconstruction ». Dans le rapport de Coconstruction, l'établissement ou l'organisme public partage ses responsabilités et ses opérations avec des organismes du tiers 
secteur mais, par opposition à la sous-traitance, chacun des acteurs «conserve son autonomie, ses valeurs et sa mission». La Coconstruction implique également une participation des organismes du tiers secteur à l'élaboration des politiques publiques. Les relations entre les deux types d'organisation sont également beaucoup plus formelles et feront souvent l'objet d'une entente écrite liant les deux organisations.

La typologie de Coston repose par ailleurs sur l'étude de trois paramètres principaux, à savoir : 1. l'ouverture au pluralisme institutionnel, c'est-à-dire l'ouverture de l'État à la présence du tiers secteur dans l'exercice de certaines activités de production et de gestion de services; 2. la formalisme des relations; et 3. la symétrie des rapports de pouvoir (voir schéma 1). Ces six types de rapport se retrouvent par ailleurs sur un continuum, de sorte que plus on se trouve sur la gauche du continuum, plus le rapport de pouvoir est dominé par l'État (rapport asymétrique). À l'opposé, plus on se trouve sur la droite du continuum, plus l'État est ouvert à se laisser influencer et à partager son pouvoir avec les acteurs du tiers secteur (rapport symétrique).

\section{Schéma 1 : Continuum de Proulx et coll., 2007 (adapté de J. Coston)}

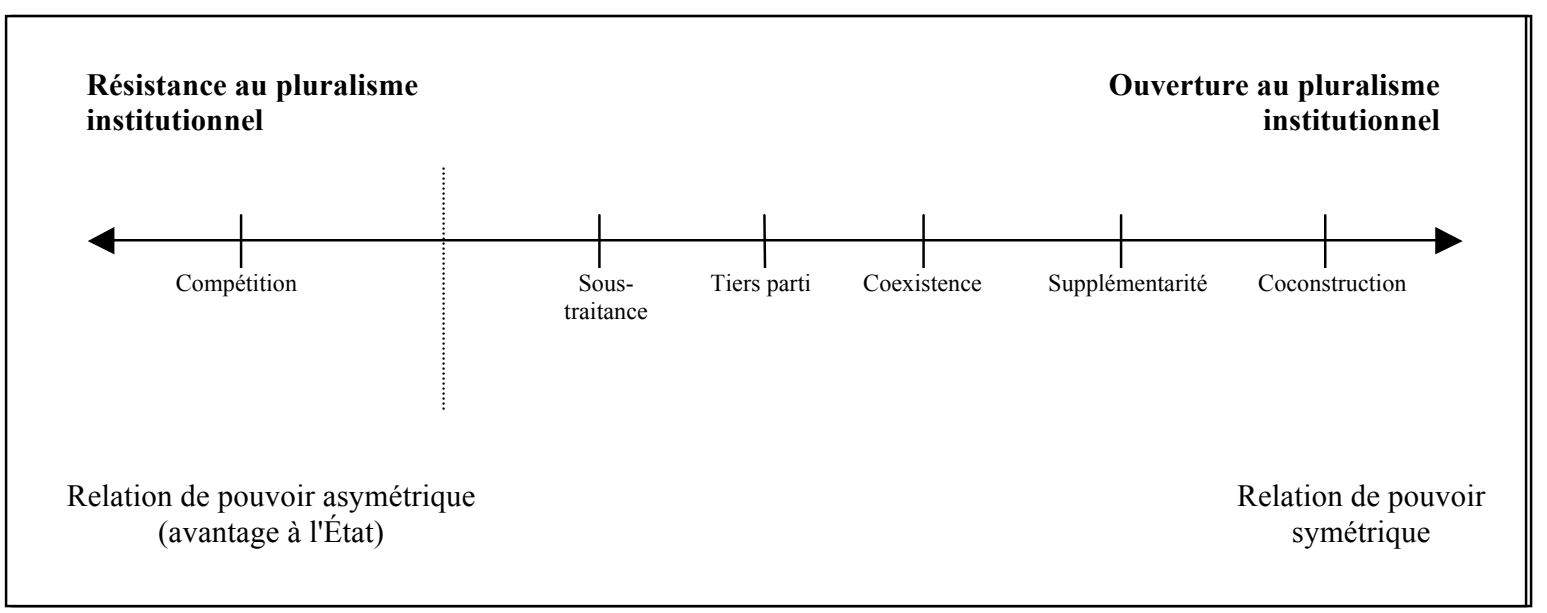

L'objectif de la recherche dont nous présentons les résultats était de deux ordres:

- Offrir aux organismes communautaires un outil favorisant l'autoévaluation et l'analyse des relations qu'ils entretiennent avec un partenaire choisi du réseau public de la santé et des services sociaux du Québec;

- Dégager, à partir de l'étude des résultats obtenus par le croisement des autoévaluations produites par les organismes participant à la validation de l'outil, les modèles de relations qui s'établissent entre les organismes communautaires et les établissements publics dans différents secteur d'intervention au Québec.

\section{MÉTHODOLOGIE DE LA RECHERCHE}

Dans le cadre de cette recherche, la première étape a consisté à élaborer un outil visant à permettre aux organismes du tiers secteur de déterminer dans quel type de rapport leur organisation se situe face à un établissement ou un organisme public avec lequel ils sont en relation (Savard et Proulx, 2011). Cet outil prend la forme d'un questionnaire auto-administrable comportant 35 questions qui peut être complété à 
l'écran. Il s'appuie sur les mêmes paramètres que ceux développés par Coston, auxquels nous en avons ajouté un quatrième, à savoir l'intensité des relations entre les deux types d'organisation. Coston n'aborde cette dimension que très succinctement. Nous avons décidé d'y donner davantage d'importance car selon nous l'intensité des relations a une influence importante sur la dynamique des rapports entre deux partenaires. Les réponses aux questions sont cotées sur une échelle de type Lickert graduée de 1 à 5 et, en fonction du résultat obtenu pour chacun des paramètres à l'étude, permettent au répondant de déterminer le type de rapport dans lequel il se situe relatif à un établissement ou un organisme public donné3.

Cette recherche s'est par ailleurs inscrite dans un exercice qui consistait à valider l'outil d'analyse des rapports à l'État. Pour procéder à cette validation, il a été estimé qu'une cinquantaine d'organismes serait suffisante étant donné que notre objectif n'était pas tant de généraliser des résultats à l'ensemble des organismes communautaires du Québec mais bien de dégager des pistes de réflexion à partir de portraits de relations issus de la perception de gestionnaires d'organismes communautaires. Par ailleurs, il apparaissait important que cette validation puisse se faire auprès d'organismes intervenant dans des secteurs d'activité diversifiés. Nous avons donc ciblé onze secteurs d'activité différents, dont huit dans le domaine de la santé et des services sociaux, à savoir :

- Les centres d'action bénévole;

- Les organismes intervenant dans le domaine de la déficience intellectuelle;

- Les organismes intervenant dans le domaine de la santé mentale;

- Les organismes intervenant auprès des familles;

- Les maisons des jeunes;

- Les organismes de justice alternative (jeunes contrevenants);

- Les organismes de travail de rue;

- Les maisons d'hébergement pour femmes victimes de violence conjugale.

Les trois autres secteurs ciblés ont été les Carrefours jeunesse-emploi (CJE), les services spécialisés de main d'œuvre (SSMO) pour personnes handicapées et les entreprises d'économie sociale en aide domestique $(E E S A D)^{4}$. Pour les organismes du domaine de la santé et des services sociaux et pour les SSMO, la cible était de quatre organismes par secteur d'activité alors que le nombre de CJE et d'EESAD visé était de huit. Dans certains cas, ce nombre a été dépassé, alors qu'il n'a pas pu être atteint dans d'autres.

Pour rejoindre ces organismes, nous avons pour l'essentiel utilisé nos contacts personnels dans les regroupements provinciaux, et parfois contacté directement certains organismes. Au total, sur les 54 organismes qui ont été sollicités, 52 ont complété le questionnaire. Ces 52 organismes provenaient par ailleurs de 15 régions différentes. Bien que notre but n'ait pas été d'obtenir un échantillon probabiliste, nous avons tout de même cherché à obtenir une certaine représentativité régionale, par exemple en sollicitant un plus grand nombre d'organismes dans la région de Montréal.

Les organismes répondant au questionnaire devaient choisir un établissement ou un organisme public (et un seul) avec lequel ils désiraient analyser leur relation. Bien entendu, selon leur secteur d'activité, les organismes sont en relation avec des établissements ou des organismes publics qui peuvent être différents, mais qui peuvent aussi parfois être les mêmes. 


\section{RÉSULTATS}

Comme souligné précédemment, l'outil construit comportait quatre dimensions : l'ouverture au pluralisme institutionnel; l'intensité de la relation; le formalisme de la relation; et la symétrie des rapports de pouvoir. Voyons donc ce qui ressort pour chacune de ces dimensions.

\section{L'ouverture au pluralisme institutionnel}

L'ouverture au pluralisme institutionnel est définie comme étant « l'ouverture de l'établissement ou de l'organisme public face à l'existence d'un autre type d'organisation offrant des activités ou des services à la même catégorie de clientèle et pouvant entretenir des philosophies et des approches d'intervention différentes » (Savard et Proulx, 2011).

De l'avis des répondants, les établissements et les organismes publics semblent démontrer une assez grande ouverture face aux organismes du tiers secteur. Le Tableau 1 présente les résultats pour quelques indicateurs.

D'abord, on constate que les directions d'établissements ou d'organismes publics démontrent une assez bonne connaissance des organismes du tiers secteur. En effet, $77 \%$ des répondants jugent que la direction de l'établissement ou de l'organisme public avec lequel ils sont en relation connaît plutôt bien, bien ou très bien la nature des activités de l'organisme. Ils sont un peu moins nombreux (64 \%) à juger que la direction de l'établissement ou de l'organisme public avec lequel ils sont en relation connaît bien, plutôt bien ou très bien leurs modes de fonctionnement et d'intervention spécifiques. Toutefois, c'est dans une proportion de 85 $\%$ qu'ils considèrent que la direction de l'établissement ou de l'organisme public avec lequel ils sont en relation respecte plutôt bien, bien ou très bien leurs modes de fonctionnement et d'intervention spécifiques.

Tableau 1 : Quelques indicateurs d'ouverture au pluralisme institutionnel

\begin{tabular}{|l|c|c|c|c|c|}
\hline & $\begin{array}{c}\text { Pas du } \\
\text { tout }\end{array}$ & Peu & $\begin{array}{c}\text { Plutôt } \\
\text { bien }\end{array}$ & Bien & $\begin{array}{c}\text { Très } \\
\text { bien }\end{array}$ \\
\hline Connaissance des activités de l'organisme & $0 \%$ & $23 \%$ & $35 \%$ & $27 \%$ & $15 \%$ \\
\hline Connaissance des modes de fonctionnement & $8 \%$ & $29 \%$ & $35 \%$ & $21 \%$ & $8 \%$ \\
\hline Respect des modes de fonctionnement & $0 \%$ & $15 \%$ & $46 \%$ & $29 \%$ & $10 \%$ \\
\hline Reconnaissance de la légitimité & $0 \%$ & $6 \%$ & $15 \%$ & $39 \%$ & $40 \%$ \\
\hline Reconnaissance de l'expertise & $0 \%$ & $12 \%$ & $37 \%$ & $35 \%$ & $17 \%$ \\
\hline & $\begin{array}{c}\text { Très } \\
\text { faible }\end{array}$ & Faible & Modérée & Grande & $\begin{array}{c}\text { Très } \\
\text { grande }\end{array}$ \\
\hline Degré d'ouverture selon les répondants & $0 \%$ & $10 \%$ & $52 \%$ & $33 \%$ & $6 \%$ \\
\hline
\end{tabular}

Par ailleurs, il apparaît clairement que la légitimité des organismes du tiers secteur, c'est-à-dire leur droit d'offrir des services et des activités dans leur champ d'intervention, ne fait aucun doute aux yeux des organismes publics. En effet, $94 \%$ des répondants au questionnaire jugent que la direction de 
l'établissement ou de l'organisme public avec lequel ils sont en relation reconnaît la légitimité de leur action, soit plutôt bien, bien ou très bien. Les répondants jugent également dans une forte proportion (89\%) que la direction de l'établissement ou de l'organisme public avec lequel ils sont en relation reconnaît l'expertise qu'ils ont développée dans leur champ d'intervention.

Enfin, la recherche montre que la majorité des établissements et des organismes publics apportent différentes formes de soutien aux organismes. En effet, $73 \%$ des organismes ayant répondu au questionnaire disent recevoir un soutien de la part de l'établissement ou de l'organisme public avec lequel ils sont en relation. Ce soutien prend la forme de prêts de locaux pour $13 \%$ d'entre eux, de soutien professionnel (incluant les prêts de personnel) pour $42 \%$ d'entre eux et de soutien financier pour $45 \%$ d'entre eux 5 . Comme l'illustre le Tableau 2, ce soutien revêt toutefois une importance variable selon les organismes.

En somme, et bien que la situation vécue par chacun des organismes ayant participé à la recherche puisse varier, les résultats tendent globalement à montrer que les établissements et les organismes publics manifestent une assez grande ouverture envers les organismes du tiers secteur. Les répondants ne sont d'ailleurs que $10 \%$ à qualifier cette ouverture de faible ou de très faible (Tableau 1). Ils sont par ailleurs $52 \%$ à la qualifier de modérée, et $39 \%$ à la qualifier de grande $(33 \%)$ ou de très grande $(6 \%)$. Ce qui semble certain par ailleurs, c'est que leur légitimité ne fait pas de doute aux yeux des établissements et des organismes publics, et que l'expertise qu'ils ont développée dans leur champ d'intervention est aussi largement reconnue.

Tableau 2 : Soutien apporté par l'organisme public

\begin{tabular}{|c|c|c|c|c|c|}
\hline & $\begin{array}{c}\text { Très } \\
\text { faible }\end{array}$ & Faible & Modérée & Grande & Très grande \\
\hline Importance accordée au soutien reçu & $18 \%$ & $16 \%$ & $24 \%$ & $22 \%$ & $20 \%$ \\
\hline & $\begin{array}{c}\text { Moins } \\
\text { de } \mathbf{1 0} \%\end{array}$ & $\begin{array}{c}\mathbf{1 0} \%- \\
\mathbf{2 4} \%\end{array}$ & $\begin{array}{c}\mathbf{2 5} \%- \\
\mathbf{4 9} \%\end{array}$ & $\begin{array}{c}\mathbf{5 0} \%- \\
\mathbf{7 4} \%\end{array}$ & $\mathbf{7 5 \%}$ et plus \\
\hline $\begin{array}{c}\text { Proportion du soutien reçu sur le budget } \\
\text { total }\end{array}$ & $33 \%$ & $19 \%$ & $10 \%$ & $10 \%$ & $28 \%$ \\
\hline
\end{tabular}

\section{L'intensité des relations}

Nous avons défini l'intensité des relations comme étant « le nombre et la fréquence des activités, que cellesci soient formelles ou informelles, que l'organisme du tiers secteur a avec un établissement ou un organisme public » (Savard et Proulx, 2011).

Des résultats, il se dégage une intensité de relation plutôt élevée entre les organismes ayant participé à la recherche et les établissements et organismes publics. C'est ainsi que près des deux tiers des répondants (62\%) soutiennent avoir au moins une interaction par semaine avec l'établissement ou l'organisme public qu'ils ont choisi pour analyser la nature de leur relation. Ces interactions peuvent prendre plusieurs formes.

Ainsi, comme l'illustre le Tableau 3, $44 \%$ des répondants affirment communiquer souvent ou très souvent de l'information concernant un usager à l'établissement ou à l'organisme public avec lequel ils sont en relation. C'est dans des proportions semblables (46\%) que l'établissement ou l'organisme public communique souvent ou très souvent à l'organisme de l'information concernant un usager. Dans le même ordre d'idée, 79 $\%$ des organismes affirment avoir eu au moins une discussion de cas avec un intervenant de l'établissement 
ou de l'organisme public avec lequel ils sont en relation au cours des trois derniers mois. Ils en ont eu entre une et trois pour la majorité d'entre eux, mais $21 \%$ des organismes disent en avoir eu au moins onze. En ce qui a trait aux références entre les deux organisations, très peu d'organismes, qu'ils soient publics ou communautaires, disent ne jamais référer d'usagers à l'autre organisation (10\% et $12 \%$ respectivement). II est toutefois intéressant de noter que, selon les répondants, c'est dans une proportion qui va du simple au double que les établissements publics réfèrent souvent ou très souvent des usagers aux organismes communautaires (59\% contre $24 \%$ ).

Enfin, un autre indicateur permettant de mesurer l'intensité des relations entre les deux types d'organisation a trait à la participation conjointe à des réunions ou à des rencontres de travail. Sur cet aspect, $79 \%$ des répondants affirment avoir tenu de telles rencontres avec l'établissement ou l'organisme public avec lequel ils sont en relation au cours des trois derniers mois (Tableau 4). Plus de la moitié (56\%) disent en avoir tenues entre une et trois, tandis que $17 \%$ disent en avoir tenu entre quatre et six, et $6 \%$ entre 7 et 10 . Par ailleurs, $81 \%$ des répondants disent avoir participé, au cours des deux dernières années, à des activités ou à des projets conjoints avec l'établissement ou l'organisme public avec lequel ils sont en relation.

\section{Tableau 3 : Quelques indicateurs quant à l'intensité des relations}

\begin{tabular}{|l|l|l|l|l|l|}
\hline & Jamais & Rarement & Parfois & Souvent & $\begin{array}{l}\text { Très } \\
\text { souvent }\end{array}$ \\
\hline $\begin{array}{l}\text { L'organisme communique de l'information à } \\
\text { l'établissement }\end{array}$ & $14 \%$ & $23 \%$ & $19 \%$ & $29 \%$ & $15 \%$ \\
\hline $\begin{array}{l}\text { L'établissement communique de l'information à } \\
\text { l'organisme }\end{array}$ & $15 \%$ & $15 \%$ & $23 \%$ & $29 \%$ & $17 \%$ \\
\hline L'organisme réfère un usager à l'établissement & $12 \%$ & $17 \%$ & $48 \%$ & $14 \%$ & $10 \%$ \\
\hline L'établissement réfère un usager à l'organisme & $10 \%$ & $17 \%$ & $19 \%$ & $21 \%$ & $38 \%$ \\
\hline & Aucune & $\mathbf{1}$ à 3 & $\mathbf{4}$ à 6 & $\mathbf{7}$ à 10 & $\mathbf{1 1 ~ o u ~ + ~}$ \\
\hline $\begin{array}{l}\text { Discussions de cas au cours des trois derniers } \\
\text { mois }\end{array}$ & $21 \%$ & $40 \%$ & $8 \%$ & $10 \%$ & $21 \%$ \\
\hline
\end{tabular}

Comme on peut le constater, les résultats montrent une intensité des relations assez élevée entre les organismes du tiers secteur et les organismes publics. Les répondants la jugent quant à eux « modérée » dans une proportion de $52 \%$, et « grande » dans une proportion de $33 \%$. Ils ne sont que $10 \%$ à la juger faible ou très faible.

\section{Tableau 4 : Participation à des réunions ou des activités conjointes}

\begin{tabular}{|c|c|c|c|c|c|}
\hline & Aucune & 1 à 3 & 4 à 6 & 7 à 10 & 11 ou + \\
\hline \multirow[t]{2}{*}{$\begin{array}{l}\text { Réunions ou rencontres de travail au cours des } \\
\text { trois derniers mois }\end{array}$} & $21 \%$ & $56 \%$ & $17 \%$ & $6 \%$ & $0 \%$ \\
\hline & Aucune & Une & Deux & Trois & 4 ou + \\
\hline \multirow[t]{2}{*}{$\begin{array}{l}\text { Activités ou projets conjoints au cours des deux } \\
\text { dernières années }\end{array}$} & $19 \%$ & $27 \%$ & $29 \%$ & $10 \%$ & $15 \%$ \\
\hline & $\begin{array}{c}\text { Très } \\
\text { faible }\end{array}$ & Faible & Modérée & Grande & $\begin{array}{l}\text { Très } \\
\text { grande }\end{array}$ \\
\hline $\begin{array}{l}\text { Degré d'intensité des relations selon les } \\
\text { répondants }\end{array}$ & $6 \%$ & $4 \%$ & $52 \%$ & $33 \%$ & $6 \%$ \\
\hline
\end{tabular}




\section{Le formalisme des relations}

Le degré de formalisme des relations est défini par « la présence ou non de structures de concertation, de mécanismes de liaison ou de collaboration (tables de concertation, lieux d'échange, comités de travail, comités cliniques, etc.) entre les deux organisations, qui peuvent être plus ou moins permanentes, ou encore par la présence ou non d'ententes, de contrats ou de protocoles liant les deux organisations " (Savard et Proulx, 2011).

À cet égard, les réponses données par les répondants montrent globalement des relations qui ont un caractère davantage formel qu'informel. En effet, $41 \%$ des répondants soutiennent entretenir des relations le plus souvent formelles (33\%) ou toujours formelles (8\%) avec l'établissement ou l'organisme public avec lequel ils sont en relation. À l'inverse, seulement $17 \%$ des répondants disent entretenir des relations le plus souvent informelles ou toujours informelles. Par ailleurs, $42 \%$ des organismes disent entretenir des relations qui sont à la fois formelles et informelles. Le Tableau 5 illustre le type de mécanisme de liaison qui existe entre les organismes du tiers secteur et les organismes publics.

\section{Tableau 5 : Mécanismes de liaison entre les organismes du tiers secteur et les organismes publics}

\begin{tabular}{|l|r|}
\hline \multicolumn{1}{|c|}{ Type de mécanisme de liaison } & $\mathbf{\%}$ \\
\hline Aucun mécanisme de liaison & $8 \%$ \\
\hline Échanges surtout de façon informelle & $21 \%$ \\
\hline Comité de concertation qui ne se réunit qu'au besoin (« ad hoc ») & $12 \%$ \\
\hline Un ou des comités qui se réunissent de façon régulière sur des objets spécifiques & $48 \%$ \\
\hline Comité de concertation permanent qui se réunit de façon statutaire & $12 \%$ \\
\hline
\end{tabular}

Ainsi, on constate que $60 \%$ des répondants (31 organismes) disent avoir un ou des comités avec l'établissement ou l'organisme public avec lequel ils sont en relation qui se réunissent de façon régulière. Pour $12 \%$ d'entre eux, il s'agit d'un comité de concertation permanent qui se réunit de façon statutaire. En revanche, $21 \%$ des organismes disent échanger surtout de façon informelle, tandis que $8 \%$ disent n'avoir aucun mécanisme de liaison avec l'établissement ou l'organisme public avec lequel ils sont en relation. Pour les organismes qui ont un comité de concertation, qu'il soit permanent ou ponctuel, la majorité (63\%) affirme s'être réunis entre trois et sept fois au cours de l'année précédente, tandis que $16 \%$ disent s'être réunis au moins huit fois.

\section{Tableau 6 : Entente ou protocole entre les organismes du tiers secteur et les organismes publics}

\begin{tabular}{|l|c|}
\hline \multicolumn{1}{|c|}{ Type d'entente ou de protocole } & $\mathbf{\%}$ \\
\hline Se limite à définir les modalités de collaboration & $3 \%$ \\
\hline $\begin{array}{l}\text { Précise les rôles et responsabilités des deux organisations, mais sans contrepartie } \\
\text { financière (entente de collaboration) }\end{array}$ & $43 \%$ \\
\hline $\begin{array}{l}\text { Précise les rôles et responsabilités attendus de l'organisme du tiers secteur et les } \\
\text { modalités de financement (entente de services) }\end{array}$ & $52 \%$ \\
\hline $\begin{array}{l}\text { Précise que l'organisme est tenu de réserver un certain nombre de places à des } \\
\text { personnes référées par l'organisme public }\end{array}$ & $3 \%$ \\
\hline
\end{tabular}


Par ailleurs, pour $77 \%$ des organismes (40 organismes), leur relation avec l'établissement ou l'organisme public est encadrée par une forme d'entente ou de protocole. Pour $3 \%$ d'entre eux (Tableau 6), cette entente se limite à définir les modalités de collaboration entre les deux organisations (par exemple, échange d'informations, références, fréquence des rencontres d'échange et de concertation, etc.). Mais pour les autres, cette entente a un caractère plus formel. Pour $43 \%$ d'entre eux, l'entente précise les rôles et les responsabilités de chacune des deux organisations eu égard à leur clientèle commune, mais sans contrepartie financière (entente de collaboration). Pour $52 \%$ d'entre eux, il s'agit d'une entente de services en bonne et due forme, c'est-à-dire qu'elle précise les rôles et les responsabilités de l'organisme communautaire eu égard à leur clientèle commune, ainsi que les modalités de financement qui sont rattachées à cette prestation de services. Ce dernier résultat est important car il fait ressortir une utilisation des contrats de services beaucoup plus importante que celle que les recherches antérieures avaient dégagée. Est-ce parce que les ententes de services gagnent en importance et en popularité? Est-ce que cette différence s'explique par l'utilisation d'un échantillon non représentatif dans notre recherche? II sera intéressant de vérifier ce phénomène dans des recherches ultérieures.

Sur ces 40 organismes qui ont une forme ou une autre d'entente avec l'établissement public, celle-ci concerne l'ensemble des activités de l'organisme ou une partie importante de celles-ci pour $78 \%$ d'entre eux. Pour les autres $(22 \%)$, elle ne concerne qu'une partie peu importante des activités de l'organisme.

Dans l'ensemble, on peut conclure à des relations plutôt formelles entre les organismes du tiers secteur et les établissements et organismes publics, même si des relations plus informelles existent aussi. Les deux tiers des répondants évaluent quant à eux le degré de formalisme de leur relation de modéré (43\%) à élevé (24\%). Six organismes seulement le jugent très élevé $(6 \%)$, tandis que $28 \%$ le jugent faible (18\%) ou très faible $(10 \%)$. Par ailleurs, il est intéressant de noter que le fait d'avoir une entente ou un protocole transcende tous les secteurs d'activités, bien que, comme on pouvait s'y attendre, les SSMO, les CJE et les EESAD soient proportionnellement plus nombreux à avoir une telle entente.

\section{La symétrie des rapports de pouvoir}

La symétrie des rapports de pouvoir est définie comme étant « la capacité des organismes du tiers secteur et des établissements et organismes publics à influencer et à déterminer la nature et l'orientation du partenariat de même que des services offerts à une population commune. Lorsque les deux types d'organisation ont une capacité d'influence et de prise de décision égale, on peut parler d'un type de relation symétrique » (Savard et Proulx, 2011).

Tableau 7 : Perception de l'influence exercée face aux organismes publics

\begin{tabular}{|l|c|c|c|c|c|}
\hline & $\begin{array}{c}\text { Très } \\
\text { faible }\end{array}$ & Faible & Modérée & Forte & Très forte \\
\hline $\begin{array}{l}\text { Capacité des organismes du tiers secteur à } \\
\text { influencer les décisions lors de réunions }\end{array}$ & $15 \%$ & $8 \%$ & $44 \%$ & $31 \%$ & $2 \%$ \\
\hline $\begin{array}{l}\text { Capacité des organismes publics à influencer } \\
\text { les décisions lors de réunions }\end{array}$ & $0 \%$ & $10 \%$ & $35 \%$ & $48 \%$ & $8 \%$ \\
\hline
\end{tabular}

Or, lorsque les répondants au questionnaire participent à des réunions ou à des rencontres de travail avec l'établissement ou l'organisme public avec lequel ils sont en relation, ou encore à des activités ou à des projets conjoints, ils jugent, pour $75 \%$ d'entre eux, qu'ils exercent une influence forte (31\%) ou modérée (44 
$\%$ ). Ils jugent toutefois que l'établissement ou l'organisme public avec lequel ils sont en relation a, dans de telles circonstances, une influence tout aussi forte (35\%) ou modérée (48\%), et même que celui-ci possède en fait un léger avantage ( $83 \%$ contre $75 \%)$. Moins du quart des répondants jugent leur influence faible (8 $\%)$ ou très faible $(15 \%)$.

II semble par ailleurs qu'une bonne proportion des organismes souhaiterait qu'on les consulte plus souvent lors de la planification de politiques, de programmes ou de plans d'action touchant leur clientèle commune (Tableau 8). En effet, un peu plus de la moitié des répondants soutiennent que l'établissement ou l'organisme public avec lequel ils sont en relation ne les consulte jamais (17\%) ou rarement (39\%). À l'opposé, $45 \%$ soutiennent que l'établissement ou l'organisme public les consulte. Par ailleurs, lorsqu'ils sont consultés sur de telles politiques ou programmes, les organismes se disent nombreux à participer. Selon les répondants, ils le font dans une proportion de $58 \%$. Leur influence en de telles circonstances apparaît cependant mitigée. En effet, $56 \%$ d'entre eux estiment qu'ils influencent rarement (48\%) ou jamais (8\%) la nature des programmes ou des politiques sur lesquels ils sont consultés. En revanche, $44 \%$ des répondants disent qu'ils les influencent souvent (25\%), très souvent ( $17 \%$ ) ou toujours $(2 \%)$.

\section{Tableau 8 : Consultation et influence sur les programmes et les politiques}

\begin{tabular}{|l|c|c|c|c|c|}
\hline & Jamais & $\begin{array}{c}\text { Rarem } \\
\text { ent }\end{array}$ & Souvent & $\begin{array}{c}\text { Très } \\
\text { souvent }\end{array}$ & Toujours \\
\hline $\begin{array}{l}\text { Lors de la planification de programmes ou de } \\
\text { politiques, l'organisme public consulte }\end{array}$ & $17 \%$ & $39 \%$ & $33 \%$ & $8 \%$ & $4 \%$ \\
\hline $\begin{array}{l}\text { Lors de telles planifications, l'organisme du } \\
\text { tiers secteur participe }\end{array}$ & $10 \%$ & $33 \%$ & $23 \%$ & $25 \%$ & $10 \%$ \\
\hline $\begin{array}{l}\text { Lors de telles planifications, l'organisme du } \\
\text { tiers secteur influence }\end{array}$ & $8 \%$ & $48 \%$ & $25 \%$ & $17 \%$ & $2 \%$ \\
\hline
\end{tabular}

Globalement, les résultats semblent montrer que les organismes du tiers secteur exercent une certaine influence dans leurs relations aux établissements et aux organismes publics, même si elle apparaît plus faible lorsqu'il est question d'influencer les politiques ou les programmes. Malgré cela, lorsqu'ils sont interrogés sur le degré de symétrie de leur rapport de pouvoir avec l'établissement ou l'organisme public avec lequel ils sont en relation, ils sont beaucoup plus nombreux à le juger faible (42\%) ou très faible (10\%) qu'à le juger élevé ( $17 \%$ ) ou très élevé $(0 \%)$. Ils sont par ailleurs $30 \%$ à le juger modéré.

\section{Les types de rapport aux établissements et organismes publics}

Enfin, au terme de l'exercice, les répondants étaient invités à identifier, en fonction de leurs réponses aux questions, dans quel type de rapport leur organisme se situait face à l'établissement ou l'organisme public choisi pour faire l'exercice. Le Tableau 9 présente un sommaire des résultats obtenus par les répondants au questionnaire.

Ainsi, on constate d'abord que la moitié des répondants en sont arrivés à un rapport de supplémentarité. Ce résultat n'apparaît pas étonnant dans la mesure où le rapport de supplémentarité, par définition, correspond à bien des égards à la réalité des organismes du secteur de la santé et des services sociaux financés dans le cadre du programme SOC : une réponse "supplémentaire " aux services publics, déterminée par les usagers eux-mêmes (services autogérés) et soutenue par la communauté, mais pour laquelle l'État en reconnaît la légitimité et accepte de la soutenir en partie (Young, 2000). II est par ailleurs intéressant de constater que ce ne sont pas seulement des organismes du secteur de la santé et des services sociaux qui 
ont conclu à un rapport de supplémentarité. En effet, on retrouve également, notamment, trois SSMO pour personnes handicapées et trois Carrefours jeunesse-emploi.

Tableau 9 : Résultat obtenu quant au type de rapport à l'organisme public

\begin{tabular}{|l|c|c|}
\hline \multicolumn{1}{|c|}{ Type de rapport } & Nombre $^{\mathbf{6}}$ & $\mathbf{\%}$ \\
\hline Compétition & 0 & 0 \\
\hline Sous-traitance & 3 & $9 \%$ \\
\hline Tiers parti & 1 & $3 \%$ \\
\hline Coexistence & 2 & $6 \%$ \\
\hline Supplémentarité & 17 & $52 \%$ \\
\hline Coconstruction & 4 & $12 \%$ \\
\hline Hybride Supplémentarité/coexistence & 4 & $12 \%$ \\
\hline Hybride Supplémentarité/Coconstruction & 1 & $3 \%$ \\
\hline Hybride Compétition/coexistence & 1 & $3 \%$ \\
\hline TOTAL & $\mathbf{3 3}$ & $\mathbf{1 0 0} \%$ \\
\hline
\end{tabular}

Par ailleurs, quatre organismes ont conclu à un rapport de coconstruction. II s'agit d'un organisme intervenant en déficience intellectuelle (en relation avec le centre de réadaptation), d'un organisme intervenant auprès des jeunes contrevenants (en relation avec le centre jeunesse), d'un organisme de travail de rue (en relation avec la municipalité) et d'une EESAD (en relation avec le CSSS). Comme on peut le constater, il s'agit d'organismes très différents les uns des autres, ce qui laisse à penser que ce type de rapport transcende les secteurs d'intervention et qu'il tient sans doute davantage aux acteurs en place qui ont su, avec le temps, établir cette relation relativement égalitaire et empreinte de réciprocité. En plus de ces quatre organismes, on peut noter aussi qu'un autre organisme est arrivé à un rapport « hybride » se situant à mi-chemin entre le rapport de supplémentarité et le rapport de Coconstruction.

Un fait qui peut surprendre est que, sur les 33 questionnaires valides, seulement trois répondants ont identifié un rapport de sous-traitance, soient deux Carrefours jeunesse-emploi et une EESAD. On peut noter enfin que nous n'avons trouvé que deux rapports de coexistence, un seul rapport de tiers parti et aucun rapport de compétition.

En somme, on constate que, dans le secteur de la santé et des services sociaux (en excluant ici les EESAD), on se situe, dans une large mesure, dans un rapport de supplémentarité avec les établissements publics. On trouvera par ailleurs un rapport de Coconstruction dans les cas où les organismes ont développé une relation plus intense et plus formelle avec l'établissement public, tout en conservant un rapport de pouvoir élevé face à l'établissement public. À l'inverse, lorsque la relation est moins intense et moins formelle, on trouvera un rapport de coexistence (deux cas) ou un rapport « hybride » situé à mi-chemin entre le rapport de coexistence et le rapport de supplémentarité (quatre cas). II est par ailleurs intéressant de constater que le rapport de supplémentarité n'est pas l'apanage des organismes du secteur de la santé et des services sociaux. Comme nous l'avons vu, on le retrouve également chez les SSMO pour personnes handicapées, chez les Carrefours Jeunesse-emploi et chez les EESAD. 


\section{DISCUSSION}

Les résultats de cette recherche tranchent de façon importante avec les études qui ont été menées dans les années 1990, et viennent confirmer, dans une bonne mesure, les recherches menées après 2003.

En effet, les études réalisées dans les années 1990 montraient des relations difficiles et tendues entre les organismes communautaires et les établissements publics, des relations dans lesquelles les organismes communautaires se sentaient peu respectés dans leur identité et dans leurs façons de faire. On peut penser que cette attitude de la part des établissements et des organismes publics à l'égard des organismes communautaires tenait en bonne partie à une méconnaissance de ces nouveaux acteurs avec lesquels ils devaient dorénavant collaborer. Or, nous avons vu dans cette étude que les établissements publics connaissent beaucoup mieux aujourd'hui les organismes communautaires, et c'est dans une proportion de $85 \%$ que ceux-ci disent se sentir respectés dans leurs modes de fonctionnement par les établissements et les organismes publics. Fait sans doute encore plus significatif, nous avons vu comment, de l'avis des répondants, leur légitimité n'est plus aujourd'hui mise en doute et comment on reconnaît l'expertise qu'ils ont développée dans leurs domaines d'intervention.

L'étude montre également à quel point les échanges ont augmenté entre les organismes communautaires et les établissements publics. À l'évidence, ces échanges se sont également beaucoup formalisés. En effet, non seulement les organismes communautaires participent-ils massivement à différents comités ou instances de concertation avec les organismes publics, mais pas moins de $75 \%$ des organismes ayant participé à l'étude disent avoir une forme ou une autre d'entente ou de protocole avec un organisme public. Mais cette formalisation des rapports ne se traduit pas forcément, comme nous l'avons vu, par des rapports de sous-traitance. Au contraire, il semble bien que, de façon générale, nous soyons bien loin ici d'une instrumentalisation des organismes communautaires par les établissements publics.

En somme, ce qui ressort de cette étude, c'est que les organismes communautaires semblent jouir aujourd'hui d'une beaucoup plus grande reconnaissance de la part des établissements et des organismes publics, une reconnaissance qui se traduit par un plus grand respect de leur identité et de leur contribution. Contrairement à ce qui pouvait se passer dans les années 1990 où, selon l'avis de bon nombre d'établissements publics, les organismes communautaires arrivaient un peu de nulle part et apparaissaient un peu comme des anachronismes dans le paysage, on voit que les organismes communautaires ont aujourd'hui pris leur place et qu'ils sont davantage respectés comme tels.

Cette plus grande reconnaissance se traduit également par des rapports qui apparaissent beaucoup plus égalitaires. Nous avons vu en effet que c'est dans une forte proportion que les organismes communautaires considèrent avoir une certaine influence auprès des organismes publics dans le cadre de leurs rapports, même si, selon eux, celle-ci apparaît plus faible lorsqu'il s'agit d'influencer les politiques ou les programmes touchant les communautés qu'ils desservent conjointement. À cet égard, il est intéressant de noter qu'il est bien possible que les organismes communautaires sous-estiment l'influence qu'ils exercent auprès des établissements publics. En effet, lorsqu'ils sont interrogés à ce sujet, cette influence se situerait, selon les répondants, entre faible et modérée, ce qui ne correspond pas aux réponses qu'ils ont fournies aux questions traitant de cet aspect. Cette perception ne se reflète pas non plus dans les résultats quant au type de rapport qu'ils entretiennent, globalement, avec les établissements et les organismes publics. En effet, un faible degré de symétrie des rapports de pouvoir, combiné aux autres dimensions à l'étude, nous conduirait vers des rapports soit de compétition, soit de sous-traitance, soit de coexistence. Or, comme nous l'avons $\mathrm{vu}$, ces trois types de rapport ne sont pas beaucoup ressortis au final. Les recherches de Savard et coll. 
(2008 et 2010) tendent également à montrer que les organismes communautaires sous-estiment l'influence qu'lls exercent auprès des établissements publics, ces derniers ayant une perception beaucoup plus positive de l'influence qu'exercent les organismes communautaires sur eux.

En somme, pris globalement, le rapport de supplémentarité, fortement majoritaire dans les résultats, reflète sans doute assez bien la tendance générale, à tout le moins pour les organismes intervenant dans le domaine de la santé et des services sociaux. Le fait que nous ayons trouvé très peu de rapports de soustraitance témoigne sans doute d'un type de relation particulier qui, avec les années, s'est installé au Québec entre les organismes communautaires et les instances publiques, un type de relation marqué par une reconnaissance réelle de l'apport des organismes communautaires dans leur champ d'intervention, de leur légitimité en tant qu'acteur (les résultats sont plutôt éloquents à cet égard) et par une type de rapport qui, sans être symétrique, ne s'inscrit pas pour autant dans l'asservissement et dans le pur utilitarisme, mais dans une certaine réciprocité.

C'est d'ailleurs en substance ce qui ressort de l'étude de Chartrand et coll., qui ont comparé le mode de relation entre l'État et les organismes du tiers secteur entre le Québec et la Saskatchewan. Ainsi, en Saskatchewan, le rapport serait caractérisé par une faible reconnaissance des organismes du tiers secteur ainsi que par la sous-traitance, contrairement au Québec où les organismes du tiers secteur jouiraient d'une forte reconnaissance et où le rapport serait également plus institutionnalisé dans un modèle qui se rapprocherait davantage de la « coproduction ». Le « modèle » québécois se démarquerait également de ce que l'on retrouve en Ontario, en Alberta et au Nouveau-Brunswick, où le rapport serait davantage empreint d'une "logique marchande ou quasi-marchande " et où c'est le bénévolat qui serait valorisé dans une " perception caritative » des organismes du tiers secteur (Chartrand et al. 2005, pp. 34-35). On peut penser que, dans le secteur de la santé et des services sociaux à tout le moins, l'augmentation importante du financement accordé aux organismes communautaires dans le cadre du programme SOC au cours des ans les a rendus moins vulnérables aux ententes de services. Jetté soutient d'ailleurs que le programme SOC constitue "une pièce maîtresse du modèle québécois de développement social» en ce qu'il permet justement aux organismes communautaires « de se prémunir, jusqu'à un certain point, contre les dérives tutélaires de l'État » (Jetté, 2008, p. 354).

Par ailleurs, si le rapport de supplémentarité représente plus de la moitié des types de rapport qui sont ressortis de cette recherche, on doit constater aussi qu'une variété de types de rapport existe au Québec. En fait, si le rapport de supplémentarité constitue sans doute le type dominant chez les organismes du secteur de la santé et des services sociaux, la situation se présente différemment dans les autres secteurs étudiés. D'ailleurs, dans le seul secteur du développement de l'employabilité, dans lequel plus de 300 organismes interviendraient au Québec, Shields soutient qu'il existe différents types de rapport aux organismes publics, une variété qui tiendrait, en partie du moins, à la diversité des cadres de financement (Shields, 2006, p. 36).

\section{CONCLUSION}

Dans cet article, nous avons présenté les résultats d'une recherche réalisée à partir de données colligées dans le contexte de la validation d'un outil créé dans l'optique de permettre à des représentants d'organismes communautaires d'analyser et d'évaluer les relations qu'ils entretiennent avec un partenaire du secteur public. À partir des réponses fournies par 52 représentants d'organismes communautaires évoluant dans différents secteurs d'intervention des services publics, nous avons pu faire un certain nombre de constatations. 


\section{Savard et Proulx (2012)}

Nous avons pu établir que les relations entre les acteurs du secteur public et du secteur communautaire semblent s'être améliorées depuis 25 ans, époque où les premières études sur les relations entre les deux groupes ont commencé à abonder au Québec. II semble en effet que les organismes communautaires ont gagné leurs lettres de noblesse, car leur importance, leur expertise et leur légitimité sont maintenant chose entendue. La culture de la concertation paraît bien établie dans les communautés, et le respect mutuel, sans être immuable, est généralement présent lorsque les établissements publics et les organismes communautaires doivent collaborer. Sans que l'on puisse qualifier nécessairement les rapports d'égalitaire, la reconnaissance du milieu communautaire passe également par une contribution et une capacité d'influences importantes sur les paramètres qui vont définir les ententes avec les établissements publics. II semble par contre que les organismes communautaires soient davantage reconnus pour leur compétence et leur indispensabilité dans la fourniture de services directs à la population, et moins comme des observateurs crédibles ou des experts pouvant contribuer à l'élaboration de politiques publiques s'adressant à un ensemble qui dépasse leur clientèle immédiate. Les expériences de coconstruction des politiques entourant la création des centres de la petite enfance et des entreprises d'économie sociale en aide domestique relèveraient donc davantage de l'exception que de la règle.

Ainsi, les résultats suggèrent que, depuis l'adoption de la Loi 120 jusqu'à aujourd'hui, les organismes communautaires, ceux du domaine de la santé et des services sociaux à tout le moins, sont passés de la coexistence à la supplémentarité. II faut cependant rappeler que l'échantillon à partir duquel les résultats ont été obtenus n'était que probabiliste. De ce fait, nous ne pouvons prétendre à une représentativité statistique. II faut donc comprendre que les résultats sont davantage des pistes de réflexion qui demanderaient à être validées ou nuancées par d'autres recherches réalisées à partir d'échantillons probabilistes aléatoires ou empiriques. II serait ainsi intéressant de pouvoir reprendre l'exercice avec l'outil auquel nous avons maintenant apporté les corrections nécessaires, mais avec un plus large échantillon d'organismes afin de pouvoir mettre en évidence une plus grande variété de types de rapports qui, selon les secteurs d'intervention, présenteraient sans doute des caractéristiques particulières. II serait également éclairant de mener une recherche avec un outil similaire, mais cette fois auprès d'un échantillon composé de représentants d'établissements publics évoluant dans différents secteurs d'intervention de l'action publique. Cela nous permettrait de comparer la perception des acteurs du secteur public avec ceux du milieu communautaire qui, comme nous l'avons vu, peuvent présenter des différences.

Somme toute, nous croyons que cette recherche ajoute sa contribution aux efforts menés par la communauté scientifique pour comprendre la nature et l'évolution des relations entre l'État et la société civile organisée. Les organismes communautaires sont un véhicule fondamental pour favoriser la participation citoyenne dans l'identification des besoins et la recherche de solutions adaptées aux réalités locales. II est donc important de continuer à investir ce champ de recherche qui est actuellement dans une période de transition importante.

\section{NOTES}

1. Plusieurs appellations ont cours pour désigner ce secteur d'activité régi sous la forme d'organisation à but non lucratif, et qui peut englober, selon les pays, des réalités différentes: " nonprofit sector »; " voluntary sector »; « nongovernmental organization »; " charitable association »; « third sector », etc. Aux fins des présentes, nous utiliserons l'expression "tiers secteur " pour désigner tout organisme à but non lucratif (OBNL) issu de la communauté, incluant les organismes communautaires, autonomes et, au sens large, les associations de bénévoles, les coopératives, les entreprises de l'économie sociale, etc. 
2. Les rapports public/communautaire ont été surtout étudiés dans le domaine de la santé et des services sociaux. Ce qui suit est donc limité à ce domaine et même, dans une large mesure, aux domaines des services de soutien à domicile et de la santé mentale puisque, jusqu'aux années 1990, c'est surtout dans ces deux domaines que les expériences de partenariat public/communautaire ont été vécues.

3. On trouvera le questionnaire à l'adresse suivante, www.larepps.uqam.ca, sous la rubrique « Outil ».

4. Bien que les EESAD interviennent dans le domaine de la santé et des services sociaux, nous en avons fait une catégorie à part puisqu'elles ont un historique et un mode de financement différents des organismes soutenus dans le cadre du Programme SOC.

5. À noter ici que les répondants étaient invités à cocher une seule réponse, soit celle correspondant au niveau de soutien le plus élevé. Ces données n'excluent donc pas qu'un organisme reçoive plus d'un type de soutien de la part de l'établissement ou de l'organisme public.

6. Sur cette question, sur les 52 questionnaires complétés, les résultats ont été jugés valides pour 33 d'entre eux. Seules les données des 33 questionnaires valides ont été compilées sur cet aspect du type de rapport.

\section{BIBLIOGRAPHIE / REFERENCES}

Bourque, D. (2007a). "Les partenariats dans le développement des communautés ». L'organisation communautaire. Fondements, approches et champs de pratique. S.I.d. de D. Bourque, Y. Comeau, L. Favreau et L. Fréchette. P.U.Q., 297-309.

Bourque, D. (2007b, Juin). « Ententes de services dans le Programme de soutien aux jeunes parents et nouveaux rapports public-communautaire ». Intervention, 126, 42-52.

Bourque, D. (2004, Novembre). Nouvelle donne dans les rapports entre réseau public et organismes communautaires. Cahiers du Larepps no 04-32. LAREPPS. UQAM.

Bourque, G.-L. (2000). Le modèle québécois de développement. De l'émergence au renouvellement. SteFoy : P.U.Q.

Caillouette, J., Garon, S. et Ellyson, A. (2007). « La gouvernance du secteur de la santé et des services sociaux: Redéfinition des rapports État-société civile ». Le système sociosanitaire au Québec: gouvernance, régulation et participation. S.I.d. de M. J. Fleury, M. Tremblay, H. Nguyen et L. Bordeleau. Gaëtan Morin Éditeur (451-466).

Chartrand, S., Nicol, R., Thériault, L., Tremblay, L., et Y. Vaillancourt (2005). Les interfaces tiers secteur État en Saskatchewan et au Québec dans le champ de la santé et des services sociaux. Cahiers du Larepps 05-18. LAREPPS. UQAM. Septembre.

Coston, J. M. (1998, Septembre). «A Model and Typology of Government-NGO Relationship ». Nonprofit and Voluntary Sector Quarterly, 27(2), 358-382.

Favreau, L. et G. Larose (2007). "Le développement des communautés aujourd'hui au Québec ». L'organisation communautaire. Fondements, approches et champs de pratique. S.I.d. de D. Bourque, Y. Comeau, L. Favreau et L. Fréchette. P.U.Q. 41-55.

Groulx, L.- H. (1993). Où va le modèle suédois? État-Providence et protection sociale. Édition l'Harmattan. P.U.M.

Jetté, C. (2008). Les organismes communautaires et la transformation de l'État-Providence. Trois décennies de coconstruction des politiques publiques dans le domaine de la santé et des services sociaux. P.U.Q.

Panet-Raymond, J. et D. Bourque (1991). Partenariat ou pater-nariat ? La collaboration entre établissements publics et organismes communautaires œuvrant auprès des personnes âgées. Groupe de recherche en développement communautaire. Université de Montréal. 
Proulx, J. (2011). Organismes communautaires et projets cliniques dans le domaine du handicap : Un état de la situation. Cahiers du Larepps 11-01. LAREPPS. UQAM. Janvier.

Proulx, J. (1997). Le partenariat entre l'État et les organismes communautaires dans le cadre de la Loi 120 : L'enjeu de la complémentarité. Mémoire de maîtrise. Université Laval.

Proulx, J., Bourque, D. et S. Savard (2007). «Interfaces between State and Third Sector in Quebec ». Voluntas: International Journal of Voluntary and Nonprofit Organizations, 18(3), 293-307.

Proulx, J., et N. Boudreault (2009). Pour un monde meilleur : Quand l'humain fait la différence. Rapport de recherche sur l'action communautaire en Mauricie et au Centre-du-Québec. Centre-duQuébec/Mauricie : Table régionale des organismes communautaires (TROC).

René, J.-F., et L. Gervais (2001). "La dynamique partenariale: Un état de la question. Les enjeux du partenariat aujourd'hui ». Nouvelles pratiques sociales, 14(1), 20-30.

Rosanvallon, P. (1981). La crise de l'État-Providence. Paris : Éditions du Seuil.

Savard, S., Robichaud, S., et S. Tremblay (2010). « Les modèles de relations partenariales dans le secteur du soutien à domicile québécois ». Intervention, 131. 249-260.

Savard S., Tremblay, S., et B. Harvey (2008). "Les relations de collaboration entre le secteur public et les organismes communautaires du secteur jeunesse-enfance-famille: Entre la sous-traitance et la Coconstruction », Revue Administration publique du Canada, 51(4), 569-588.

Savard, S. (2002). La concertation et la collaboration autour de la fourniture de services sociaux aux jeunes et aux familles : une analyse stratégique. Thèse de doctorat en service social déposé à la Faculté des sciences sociales. Université Laval.

Savard, S. et J. Proulx (2011). Outil d'analyse des rapports à l'État. LAREPPS. UQAM.

Shields, G. (2006). Dynamiques partenariales dans le champ de la main-d'œuvre (1996-2003). Le défi d'une nouvelle gouvernance québécoise impliquant les organismes communautaires d'insertion. Cahiers du Larepps 06-01. LAREPPS. UQAM. Janvier.

Vaillancourt, Y. (2012). « The Quebec model of social policy, past and present ». In Anne Westhues et Brian Wharf (Eds). Canadian Social Policy: Issues and Perspectives. Fifth edition. Waterloo: Wilfrid Laurier University Press.

Vaillancourt, Y., avec la collaboration de Leclerc (2008). Note de recherche sur l'apport de l'économie sociale dans la coproduction et la coconstruction des politiques publiques. Cahiers du Larepps 0801. LAREPPS. UQAM. Janvier.

Vaillancourt, Y. (1997). Vers un nouveau partage des responsabilités dans les services sociaux et de santé : Rôles de l'État, du marché, de l'économie sociale et du secteur informel. Cahiers du Larepps 9705. LAREPPS. UQAM. Janvier.

White, D., Mercier, C., Dorvil, H. et L. Juteau (1992). «Les pratiques de concertation en santé mentale : Trois modèles ». Nouvelles pratiques sociales, 5(1), 77-93.

Young, D. R. (2000). "Alternatives models of government-nonprofit sector relations: Theoretical and international perspectives ». Nonprofit and Voluntary Sector Quarterly, 29(1), 149-172.

\section{LES AUTEURS / ABOUT THE AUTHORS}

Sébastien Savard est professeur agrégé à l'École de service social de l'Université d'Ottawa. 120 rue de I'Université, Ottawa ON K1N 6N5. Courriel : Sebastien.savard@uottawa.ca

Jean Proulx est professionnel de recherche au Laboratoire de recherche sur les pratiques et politiques sociales (Larepps). École de travail social, Université du Québec à Montréal (UQAM), C.P. 8888, succ. Centre-ville, Montréal QC H3C 3P8. Courriel : Jean.proulx@sogetel.net 